\title{
REMOTE SENSING APPLICATIONS WITH HIGH RELIABILITY IN CHANGJIANG WATER RESOURCE MANAGEMENT
}

\author{
Li Ma ${ }^{1,2, *}$, Shengyi Gao ${ }^{1,2}$, Aiming Yang ${ }^{1,2}$ \\ ${ }^{1}$ Changijiang Institute of Survey, Planning, Design and Research, No. 1863 Jiefang Avenue, Wuhan, Hubei, P.R.China \\ ${ }^{2}$ Changjiang Spatial Information Technology Engineering Co., Ltd, No. 1863 Jiefang Avenue, Wuhan, Hubei, P.R.China \\ - (mali2, gaoshengyi, yangaiming)@ cjwsjy.com.cn
}

TC III, WG III/1

KEY WORDS: Remote Sensing, High Reliability, Water Resource Management, Interpretation, PIR

\begin{abstract}
:
Remote sensing technology has been widely used in many fields. But most of the applications cannot get the information with high reliability and high accuracy in large scale, especially for the applications using automatic interpretation methods. We have designed an application-oriented technology system (PIR) composed of a series of accurate interpretation techniques, which can get over $85 \%$ correctness in Water Resource Management from the view of photogrammetry and expert knowledge. The techniques compose of the spatial positioning techniques from the view of photogrammetry, the feature interpretation techniques from the view of expert knowledge, and the rationality analysis techniques from the view of data mining. Each interpreted polygon is accurate enough to be applied to the accuracy sensitive projects, such as the Three Gorge Project and the South - to - North Water Diversion Project. In this paper, we present several remote sensing applications with high reliability in Changjiang Water Resource Management,including water pollution investigation, illegal construction inspection, and water conservation monitoring, etc.
\end{abstract}

\section{INTRODUCTION}

Remote sensing technology has been widely used in many fields (Chen et al., 2015). In academia, there are many studies used to improve the level of automatic identification by computers. The research progress has been greatly promoted the progress of the remote sensing application. It seems that the level of application of remote sensing is directly related to the level of intelligent research in the computers field. This leads to two unexpected results. Firstly, due to the limitation of the currently automatic classification and automatic identification technologies, the accuracy and reliability of the automated progressed applications can hardly reach the human level. It is difficult to meet the requirements of high level Water Resource Management focused on reliability and accuracy. Secondly, a lot of studies are focused on algorithm research, ignoring that the real applications are not only the academic issues, but also the strategies and path issues. The current researches are very fewly focused on the path and strategy research of application-oriented technology, while ignoring the direct use of human experience in object identification.

We propose an application-oriented remote sensing interpreting technology system (PIR) with high reliability and high accuracy in three directions: the accuracy of spatial positioning, the accuracy of the identification of object features, the rationality of interpreting results. The system focuses mainly on the strategy design for the accuracy sensitive applications such as illegal construction monitoring near the water area,water pollution source investigation and the water conservation monitoring.

\section{METHODS}

To meet the requirements in the detailed water resource management, we design our remote sensing strategies for application from three perspectives. The application-oriented remote sensing interpretation technology system consists of three parts: space positioning technology, feature interpretation technology, and rationality analysis technology, called $\mathrm{P}$ (Positioning)I(Interpretation)R(Rationality) for short . The PIR methods are respectively aimed at the accuracy and reliability of spatial location, the accuracy of feature identification, and the rationality of the results (see Figure 1).

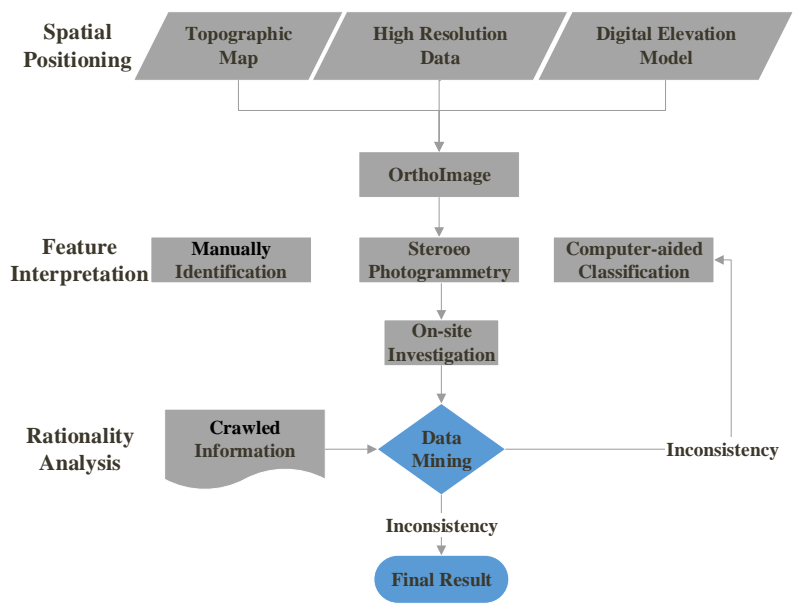

Figure 1. PIR method

\subsection{Spatial Positioning Technique}

High reliable remote sensing applications must be based on precisely processed remote sensing data. We insist that each reliability sensitive projects should be processed with high positioning accuracy. The most of usual remote sensing applications use the direct positioning of satellite or aerial remote sensing images or simple control point correction methods. The accuracy of plane positioning will reach deviations of several meters to several hundred meters (Wang et al., 2017) . The image deformation in spatial locations will seriously affect

\footnotetext{
* Corresponding Author
} 
both the measurement accuracy and the interpretation reliability (Chen et al., 2014). Especially for those applications with social sensitivity such as water resources compensation, once there is a significant deviation from the real location on the site, there will be great problems.

At present, a series of techniques in photogrammetry fields can already meet the requirements of precise positioning. For aerial or satellite imagery with a resolution of $15 \mathrm{~cm}$ to $1 \mathrm{~m}$, people can get accurately spatially corrected remote sensing images both in plane and elevation,combined with various other techniques such as image post-processing techniques.

In our remote sensing applications, we process data in strict technical standards for the ortho-rectification of remote sensing images. We suggest using the coordinate reference be consistent with the on-site measurement coordinate system to reduce the image projection deformation, not just the WGS84 latitude and longitude coordinates. We orthorectify the data by differential rectification with the help of DEM (Digital Elevation Model) data. Through the spatial positioning process, we ensure the unified coordinate reference and absolutely consistent in the accuracy of mathematics in the following procedures.

\subsection{Feature Interpretation Technique}

Feature interpenetration is often associated with automatic computer classification or the automatic identification technology, partly because of the effort to replace people's judgments or improve the computer intelligence. But we must admit that the computer has not been clever enough to fully replace the human brain in the current remote sensing reality. The reality is that in most applications, people have to takes $120 \%$ of the effort to correct $1 \%$ of errors in the computer algorithm based applications. We insist on the full use of the human knowledge.

Combined with on-site investigations, various types of remote sensing interpretation marks were established. Characteristics of different interpretation objects were carefully distinguished. In order to ensure the accuracy of Interpretation in micro-scale. We propose our feature interpretation techniques should follow three strategies.

1) On the interpreting mode, high resolution remote sensing data should be used, and all features should be manually interpreted to identify and extract features. It is due to the fact that the current level of computer development cannot yet reach the level of human decision-making.

2) On the means of interpretation, we suggest the interpretation under the environment of photogrammetry workstation (see Figure 2). We suggest the use of stereo imagery of aerial or satellite remote sensing data. From the view of photogrammetry, we recover the real-world scenario of the data acquisition. We can observe features in the stereo environment while retain all the spectral information.Not only the spectral information and plane information of the image are used, but also the height information of the ground object is fully utilized. Human beings can recognize and extract detailed features enough to ensure the accuracy and reliability though vision check.

3) On the consistency strategy in case of dynamic monitoring, we use the strategy consistent with the previous method while make necessary corrections with high resolution images. If the previous results are based on low-to-medium-resolution remote sensing data using the computer-based automatic classification method, we used the automatic computer classification method consistent with the previous period to ensure the variation trend of the features. However, we still recommend the use of highresolution stereo remote sensing images in all period if condition permits.

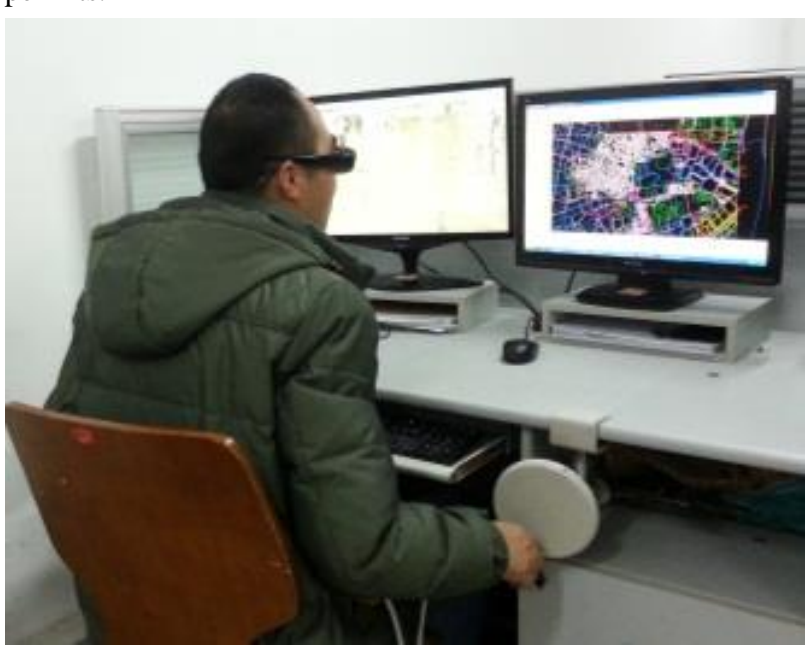

Figure 2. Photogrammetry-based interpretation

\subsection{Rationality Analysis Technique}

We believe that the status and the changes of the objects are not isolated and must be the result from the joint action of natural and human activities. In the process of providing technical support for management departments, we have established a set of rationality analysis indicators for the analysis of the rationality of the Interpretation results. Two steps are taken as follows.

The first step is the information collection through data crawling and data mining techniques. We completely collect policies formulated by management departments or local governments, the measures taken in remote sensed region, the effect of the implementation, the news report on the remote sensed object or on the effect of the activity and other relevant information.

The second step is rationality indicators computation from different dimensions. Once there is a anomaly on the data trend or the data consistency, comprehensive consultation should be carried out between the interpretation results and the analysis results. On-site re-sampling verification is necessary to ensure the reliability in some cases.

\section{APPLICATIONS}

\subsection{Water Pollution Investigation in South-to-North Water Transfer Project}

The Danjiangkou reservoir is the water source of the South-toNorth Water Transfer Project, covering an area of $1047.5 \mathrm{~km}^{2}$. The cage culture in the Danjiangkou reservoir is a challenge to the dissolved oxygen tolerance of the whole water area. The long-term deposition of fish baits and fish excreta will increase the ammonia nitrogen content in the water and affect the water quality in the reservoir area. All of the net cages must be cleaned up to ensure the water quality. In order to meet the need for the supervision of cages, satellite remote sensing monitoring techniques were used in 2013, 2016 and 2017 to monitor the cages in Danjiangkou reservoir area. 
We collected the Worldview and Beijing-2 high resolution images as our remote sensing data.The resolutions of the image are between $0.5 \mathrm{~m}$ to $0.8 \mathrm{~m}$. We rectified all the image strictly in photogrammetry workstations with the help of DEM data and the existing 1:5000 topographic maps. The coordinate system of all the images is unified to Beijing 1954 datum and Gauss projection. The accuracy of the rectified orthoimages is better than 1 meter. Interpretation marks have been established after on-site investigations. All the net cages in the image have been manually interpreted by over 10 experienced operators. Figure 3 showed one type of net cages in Danjiangkou water area.

After the finish of manually interpreted polygons,we collected several reports on the cleanup operations from the management departments and crawled the news from the government or social articles on industry adjustment in Danjiangkou area between 2013- 2017. We calculated the variation trend and compared with the statistical results of interpretation. The calculating results tally with the interpretation results. On-site re-sampling verification is shown that the interpretation accuracy reach up to $97 \%$, which meant that the extraction of net cage information was highly acceptable.

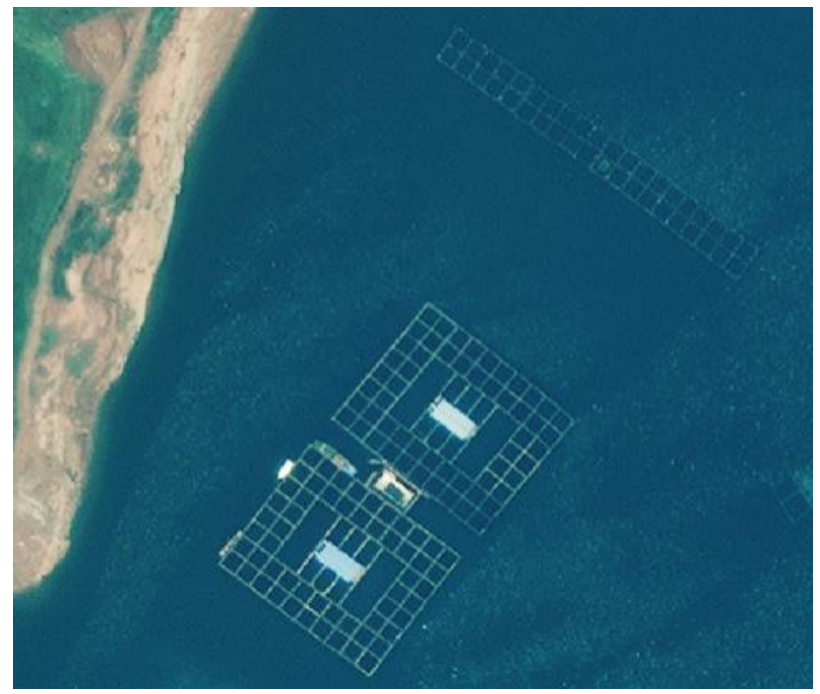

(1) Satellite image

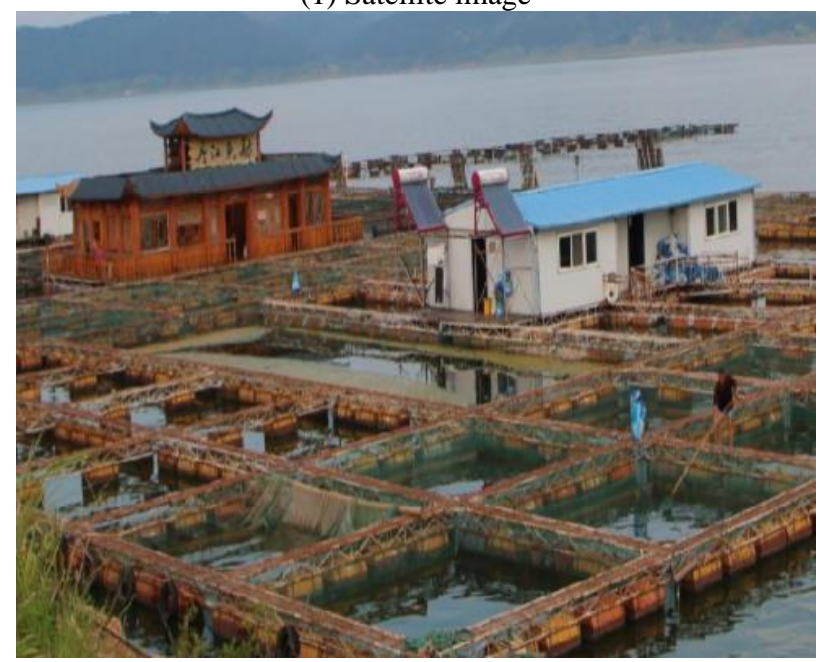

(2) On-site photo

Figure 3. One type of net cages

\subsection{Illegal Construction Inspection from Remote Sensing Data}

From 2015 to 2017, we adopted our PIR technology to monitor the behavior of damaging the ecological environment in the Three Gorges and the South-to-North Water Transfer Project, such as blocking dams, occupying storage capacity, illegally setting up docks, illegally setting up sewage outlets, and dumping of waste near the water area, covering over $10311 \mathrm{~km}$ water fronts.

The interpreted results have been used as the illegal evidence to the water law enforcement agencies. Related results are described in another article (Zhong et al., 2017). Figure 4 showed an illegal damming evidence in a secluded tributary.
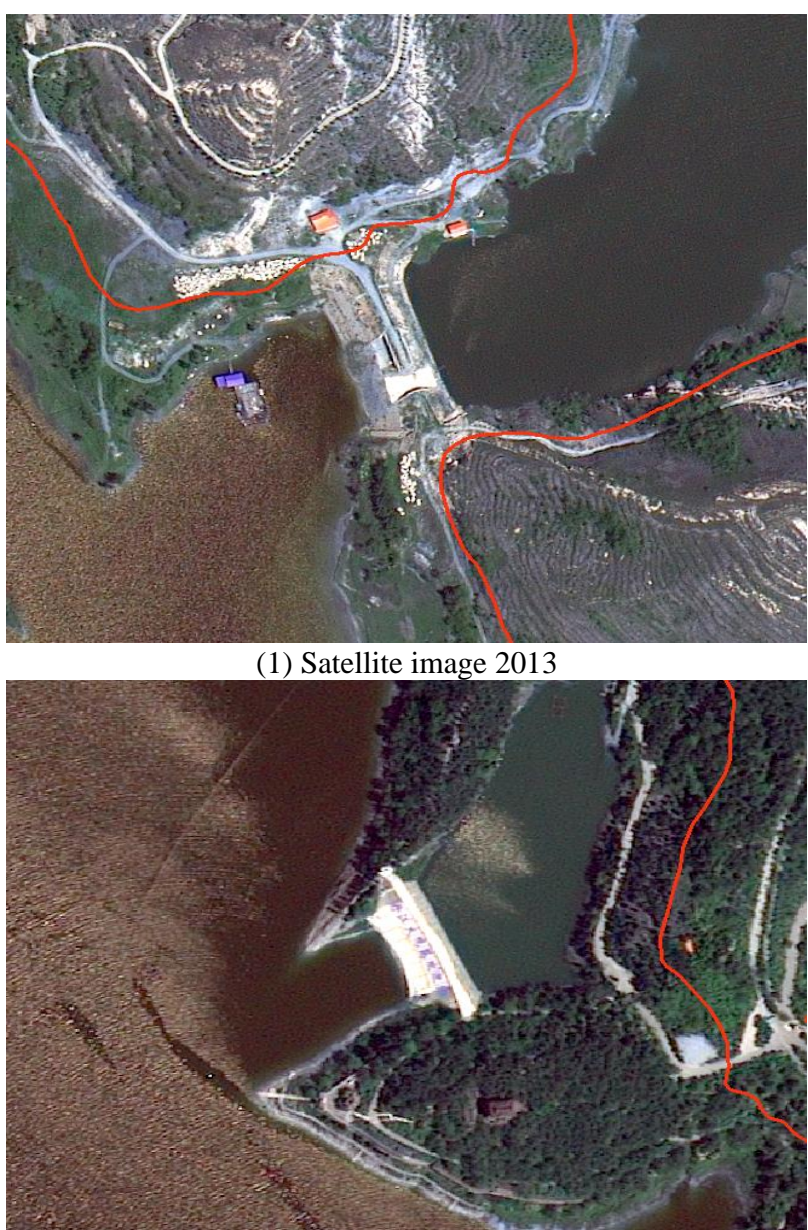

(2) Satellite image 2017

Figure 4. An illegal damming evidence

\subsection{Water Conservation Measures Monitoring}

From 2015 to 2018, we adopted our PIR technology to monitor the soil and water conservation measures in Jinsha and Southwest rivers watershed, covering an area over $100,000 \mathrm{~km}^{2}$. The area of minimum polygon is $2500 \mathrm{~m}^{2}$. Figure 5 showed the water conservation polygon interpreted

We collected the Chinese GF and ZY series 2 meters resolution images as our remote sensing data.We rectified all the image in one pixel with the help of the existing DEM data and existing field control points. The coordinate system of all the images in different years is unified to CGCS2000 datum and Gauss projection. Interpretation marks have been established after on- 
site investigations. All the soil types and water conservative measures including plant measures, engineering measure and tillage measures have been manually interpreted by experienced operators according to the technical specification (MWR, 2012).

The mined variation trend and government-invested project information was highly consistent with our statistical results of interpretation. On-site re-sampling verification is shown that the interpretation accuracy reach up to $92 \%$, which meant that the information extracted was highly reliable.

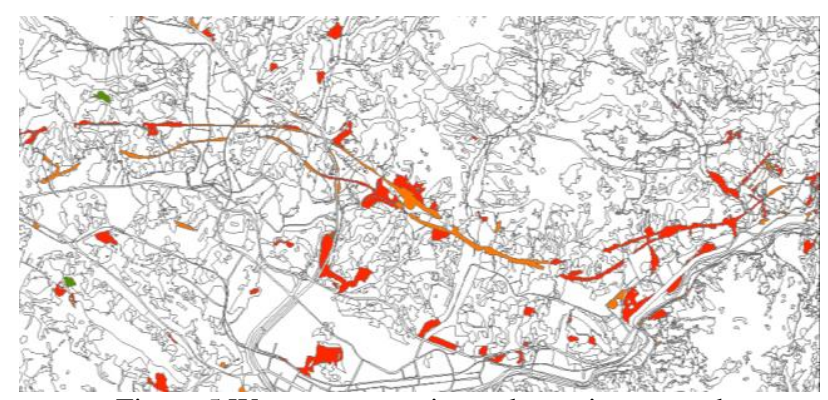

Figure 5 Water conservation polygon interpreted

\section{CONCLUSIONS}

In this paper, we introduce our PIR technology for precise remote sensing interpretation applications in water resource management. The applications in the water pollution investigation, illegal construction inspection and water conservation monitoring have shown the high reliability and high accuracy. Application-oriented remote sensing monitoring is not only an academic research issue, but also a path and strategy issue.

\section{REFERENCES}

Chen G., et al., 2014. Assessment of the image misregistration effects on object-based change detection. ISPRS Journal of Photogrammetry and Remote Sensing. Vol.87, pp.19-27.

Chen, J., et al., 2015. Information from imagery: ISPRS scientific vision and research agenda. ISPRS Journal of Photogrammetry and Remote Sensing.Vol.115, pp.3-21.

Ministry of Water Resources (MWR), 2012.Technical specification of soil and water conservation monitoring by remote sensing. SL592-2012.

Wang, R., Wang, J., Hu, X., 2017. Analysis of location accuracy without ground control points of optical satellite Imagery. Acta Geodaetica et Cartographica Sinica, 46(3), pp.332-337.

Zhong, L., Gan, Z., Li Y., et al., 2017. Applications of remote sensing techniques in law enforcement for administration of water resources. In: Water Conservancy Innovation of Remote Sensing Applications In Water Conservancy Forum,pp.128-131. 